Published in final edited form as:

Clin Hemorheol Microcirc. 2018 ; 70(1): 95-105. doi:10.3233/CH-170340.

\title{
GBT440 IMPROVES RED BLOOD CELL DEFORMABILITY AND REDUCES VISCOSITY OF SICKLE CELL BLOOD UNDER DEOXYGENATED CONDITIONS
}

\author{
Kobina Dufü ${ }^{\# 1}$, Mira Patel ${ }^{\# 1}$, Donna Oksenberg ${ }^{1}$, Pedro Cabrales ${ }^{2}$ \\ ${ }^{1}$ Global Blood Therapeutics Inc., 400 East Jamie CT, South San Francisco, CA, 94080 \\ ${ }^{2}$ Department of Bioengineering, University of California, San Diego, La Jolla, CA92093 \\ \# These authors contributed equally to this work.
}

\begin{abstract}
Background: In sickle cell disease (SCD), polymerization of hemoglobin S (HbS) leads to the formation of rigid, non-deformable sickled RBCs. Loss of RBC deformability, sickling and irreversible membrane damage causes abnormal blood rheology, and increases viscosity which contributes to vasoocclusion and other SCD pathophysiology. GBT440 is a novel antipolymerization and anti-sickling agent currently undergoing clinical evaluation for the treatment of SCD.
\end{abstract}

Objective: The purpose of this study was to determine the effects of GBT440 on deformability of sickle RBCs (SS RBCs) and the hyperviscosity of sickle cell blood (SS blood).

Methods: The mechanical and rheological properties of GBT440-treated SS RBCs were measured using micropipette and filtration techniques. The viscosity of sickle blood was measured using a Wells-Brookfield cone/plate viscometer.

Results: GBT440 restored movement of deoxygenated SS RBCs through a gel filtration column and reduced the pressure required to pass SS RBCs through a polycarbonate filter. Moreover, GBT440 decreased the membrane shear elastic modulus of SS RBCs assessed via micropipette aspiration and reduced the hyperviscosity of SS blood under deoxygenated conditions.

Conclusions: GBT440 maintains SS RBC deformability and improves SS blood viscosity by inhibiting $\mathrm{HbS}$ polymerization under deoxygenated conditions. These results further support development of GBT440 as a disease-modifying agent in SCD patients.

\section{Keywords}

Sickle cell disease; red blood cell deformability; sickling; viscosity

Correspondence: Pedro Cabrales, Ph.D., University of California, San Diego, Department of Bioengineering, 0412, 9500 Gilman Dr., La Jolla, CA 92093-0412, Telephone: (858) 534-8197, pcabrales@ucsd.edu. Author Contributions: KD wrote the manuscript; K.D., M.P.P. and P.C. designed, conducted the deformability and viscosity assays and interpreted data. DO interpreted and discussed data. All authors read and revised the manuscript. 


\section{Introduction}

The rheological and hemodynamic behavior of blood in normal and disease states is primarily determined by the biomechanical properties of red blood cells (RBCs). Sickle cell disease (SCD) is a genetic disorder characterized by abnormal blood rheology, hemolytic anemia and vasoocclusion [1]. A requisite step in the molecular pathogenesis of SCD is the polymerization of deoxygenated hemoglobin $\mathrm{S}(\mathrm{deoxy}-\mathrm{HbS})$ [2,3]. Polymerization of deoxy$\mathrm{HbS}$ leads to the formation of dense, mechanically fragile and poorly deformable sickled RBCs [1]. Red blood cell deformability has a major influence on blood rheology and hemodynamics in SCD. With a thickness of 2-3 $\mu \mathrm{m}$ and a diameter of 6-8 $\mu \mathrm{m}$, RBCs must remain deformable to efficiently travel through tissue capillaries that typically have a diameter of 5-10 $\mu \mathrm{m}$ [4]. In general, RBC deformability depends on the geometry, cytoplasmic viscosity and viscoelastic properties of the RBC membrane [4]. However, under deoxygenated conditions, sickle RBC (SS RBC) deformability is significantly driven by cytoplasmic viscosity due to HbS polymer formation $[4,5]$. The inability of sickled RBCs to deform can directly cause vasoocclusion in tissue capillaries [6]. Moreover, adhesion of sickled RBCs to the endothelium of postcapillary venules has also been proposed to initiate and propagate episodes of vasoocclusion [7]. At normal partial oxygen pressure $\left(\mathrm{pO}_{2}\right)$, whole blood from SCD patients shows minor abnormalities in microvascular flow, internal cell viscosity and membrane elasticity. On the other hand, under deoxygenated conditions, extensive HbS polymerization, resulting in sickling and irreversible membrane damage of SS RBCs leads to aggravated hyperviscosity that affects in vivo microcirculatory flow dynamics contributing to vasoocclusion and impaired oxygen delivery to tissues [8].

The exact mechanism of vasoocclusion and the interplay of the factors (including hemoglobin, hematocrit, \% $\mathrm{HbF}$, dense cells, reticulocytes and irreversible sickled cells) that modulate the frequency and severity of painful vasoocclusive crisis in SCD are not well understood $[9,10,11]$. However, changes in SS RBC deformability and the hyperviscosity of sickle blood are important contributors to vasoocclusion biology and other pathophysiology in SCD $[9,12,13,14,15]$. Thus, an agent that decreases blood hyperviscosity and modulates SS RBC deformability may be beneficial for SCD patients. GBT440 is an allosteric modulator of $\mathrm{Hb}-\mathrm{O}_{2}$ affinity. When bound to $\mathrm{HbS}$, GBT440 maintains $\mathrm{HbS}$ in the oxy-Hb state, delays in vitro $\mathrm{HbS}$ polymerization and inhibits $\mathrm{RBC}$ sickling [16].

Using RBC filterability as a surrogate for deformability, we determined the effect of GBT440 on SS RBC deformability in vitro using three well characterized biomechanical experimental systems [8] which measured 1) the movement of SS RBCs through a gel filtration column, 2) the pressure required to pass SS RBCs through a polycarbonate filter, and 3) the tension required to aspirate SS RBCs into a micropipette under deoxygenated conditions. The effect of GBT440 on the viscosity of SS blood was also evaluated using a Wells-Brookfield cone/plate viscometer. 


\section{Materials and Methods}

GBT440: (2-hydroxy-6 ((2-(1-isopropyl-1H-pyrazol-5-yl) pyridin-3-yl) methoxy) benzaldehyde). GBT440 was synthesized at Global Blood Therapeutics Inc. (South San Francisco, CA, USA). Dimethyl sulfoxide (DMSO, cat\# D128-500) was from Fisher (Pittsburgh, PA), 24-well permeable plates (cat\# 3231-20) was from Coy Laboratory Products (Grass Lake, MI). Adenine (cat\# 0183) was from Amresco. Inosine (cat\# I4125) and D-(+)-Glucose (cat\# G7021) were from Sigma Aldrich (St Louis, MO).

\subsection{Blood source}

Sickle cell disease blood was obtained in sodium citrate (3.2\% citrate) vacutainers from homozygous sickle cell patients. The study was approved by the IRB of the University of North Carolina (UNC) Sickle Cell program [IRB \# 88-0343 (GCRC-101)]. Blood from trait individuals (AS) collected in sodium citrate vacutainers was purchased from Conversant Bio, Inc. (Huntsville, AL). Blood from healthy donors (AA) collected in sodium citrate vacutainers was purchased from the Stanford Blood Center (Palo Alto, CA).

\subsection{Preparation of blood for storage}

Following phlebotomy, whole blood (SS and AA) was shipped on ice (with blood not in direct contact with ice) to Global Blood Therapeutics for processing the following day. Upon receipt, less than 24 hours post collection, blood was immediately processed by addition of adenine, inosine and glucose to a final concentration of $1 \mathrm{mM}, 10 \mathrm{mM}$ and $5 \mathrm{mM}$, respectively, and stored at $4{ }^{\circ} \mathrm{C}$. These conditions allow blood to be used up to 3 weeks [17]. Blood was used for studies within 48 hours post collection.

\subsection{Gel filtration deformability assay}

Red blood cell deformability was assessed in a modified gel filtration method originally reported by Pais E. et al. [18]. A $500 \mu \mathrm{L}$ packed S-500 sephacryl (GE Healthcare) column containing deoxygenated phosphate buffered saline (PBS) was prepared by equilibrating the resin four times with PBS $(1 \mathrm{~mL})$ pre-incubated in a humidified deoxygenated chamber (98\% nitrogen $/ 2 \% \mathrm{O}_{2}$ ) for $2 \mathrm{hr}$. Next, a suspension of RBCs in PBS was incubated with varying concentrations of GBT440 or DMSO for $1 \mathrm{hr}$ at room temperature. Following incubation, RBC suspensions were deoxygenated in a humidified deoxygenated chamber (98\% nitrogen $/ 2 \% \mathrm{O}_{2}$ ) for $30 \mathrm{~min}$ and $6 \mu \mathrm{L}$ was loaded on top of a $500 \mu \mathrm{L}$ packed $\mathrm{S}-500$ sephacryl (GE Healthcare) column containing deoxygenated PBS. The column was then centrifuged for $3 \mathrm{~min}$ at $3000 \mathrm{~g}$ in an Eppendorf $-5810 \mathrm{R}$ centrifuge at RT. After centrifugation, deformable RBCs move through the sephacryl column, whereas rigid nondeformable sickled RBCs remain on top of the sephacryl resin (Fig. 1A). Deformable RBCs were separated from non-deformable RBCs and separately mixed with $1 \mathrm{~mL}$ of water to lyse RBCs. The relative concentration of $\mathrm{Hb}$ in deformable and non-deformable $\mathrm{RBC}$ fractions was determined by measuring the optical density (OD) at $542 \mathrm{~nm}$ and $700 \mathrm{~nm}$. The absorbance of $\mathrm{Hb}$ in the deformable and non-deformable $\mathrm{RBC}$ fractions reflects the number of RBCs in each fraction. Thus, the relative absorbance of the two $\mathrm{Hb}$ fractions was used to estimate percent deformability as follows: [OD $(542 \mathrm{~nm}-700 \mathrm{~nm})$ of $\mathrm{Hb}$ in deformable 
$\mathrm{RBC}$ fraction / OD (542 nm -700 nm) of $\mathrm{Hb}$ in deformable RBC + non-deformable RBC fraction] $* 100$.

\subsection{RBC filterability}

Red blood cell deformability using the filterability method was determined as previously described [19]. Red blood cell suspensions were adjusted to $20 \%$ Hct and incubated with varying concentrations of GBT440 or DMSO for 30 min at RT in PBS containing $0.5 \%$ bovine serum albumin (BSA). Red blood cells were then deoxygenated by direct exposure to low oxygen gas, as a thin film of blood on an inclined glass plate was exposed to air in a cylindrical container (Fig. 1B). The equilibrated RBC suspension was passed through a polycarbonate filter ( $5 \mu \mathrm{m}, 13 \mathrm{~mm}$ diameter, pore density of $4 \times 10^{5} / \mathrm{cm}^{2}$, Nuclepore) at various flow rates $(0.5,0.6,0.7,0.8,0.9$ and $1.0 \mathrm{~mL} / \mathrm{min})$ and differential pressure drop across the filter was measured [BIOPAC Systems, (Fig. 1B)]. The stretching factor ( $\beta$ factor) was calculated, to quantify the resistance of a single RBC to pass through a single pore independent of cell concentration and cell to pore volume [20].

\subsection{Micropipette aspiration}

Red blood cell suspensions were adjusted to $20 \% \mathrm{Hct}$ and incubated with varying concentrations of GBT440 or DMSO for $30 \mathrm{~min}$ in PBS containing 0.5\% BSA. Red blood cells were then diluted to $0.5 \% \mathrm{Hct}$ and deoxygenated by direct exposure to low oxygen gas, as a thin film of blood on an inclined glass plate was exposed to air in a cylindrical container (Fig. 1B). The equilibrated RBC suspension was transferred to a glass chamber attached to an isothermal stage regulated to $37^{\circ} \mathrm{C}$ (Sensortec, Inc.) and low oxygen gas was continually passed on top of the samples to prevent re-oxygenation. Micropipettes (A.M. Systems) were pulled (P-97, Sutter Instruments) to an internal diameter of $\sim 2$ micron and opening angle of $\sim 9^{\circ}$. The micropipette was attached on a pneumatic micromanipulator (Narishige, Japan) and secured to an inverted microscope (Olympus IMT-2, Tokyo, Japan). Aspiration pressure was induced hydrostatically and kept constant. Measurements were done with a high magnification 100X LUMPFL (Olympus, Tokyo, Japan) objective and a video system (Cohu, Inc. San Diego, CA). Subsequent aspiration length was measured off line from recorded images. Cell membrane elastic module and cell volume were calculated based on the relationship between pressure and aspiration length as previously reported [21].

\subsection{Viscosity measurement of whole blood samples at various shear rates}

Viscosity measurements were conducted using a Wells-Brookfield Cone/Plate viscometer at shear rates ranging from $60-415 \mathrm{~s}^{-1}$. The Hct was adjusted to $30 \%$ for all blood samples to ensure that changes in viscosity were not due to changes in Hct as blood viscosity increases exponentially with Hct [22]. Blood samples were incubated with varying concentrations of GBT440 or DMSO at room temperature for 30 minutes. Healthy volunteers (AA blood) and SCD (SS blood) samples $(500 \mu \mathrm{l})$ were also run as oxygenated controls. The remaining samples $(640 \mu \mathrm{l})$ were divided into two wells and they were deoxygenated for 2 hours in gas permeable 24-well plates in a humidified incubator $\left(37^{\circ} \mathrm{C}\right)$ at $2.4 \% \mathrm{O}_{2}$. These samples were then measured in a cone/plate viscometer placed inside a deoxygenated chamber $\left(96 \% \mathrm{~N}_{2} / 4 \% \mathrm{O}_{2}\right)$. The temperature of cone/plate viscometer was set to $37^{\circ} \mathrm{C}$ and all samples were given time to equilibrate to $37^{\circ} \mathrm{C}$ before measurements were conducted. 


\subsection{Viscosity Data analysis}

Data collected was analyzed using the Rheocalc software provided with the Brookfield Cone/Plate viscometer using the Bingham plastic model, which provided a blood viscosity value $(\mathrm{cP})$ for each dataset. The Bingham plastic model is appropriate as blood has shear thinning properties (viscosity is high at low shear rates, but decreases with increasing shear rates [22]).

\subsection{Statistical analysis}

Results are presented as mean \pm standard deviation. Statistical significance for the gel filtration column, the membrane shear elastic modulus, and the blood viscosity were analyzed using one-way Kruskal-Wallis ANOVA, followed by Dunn's multiple comparisons post hoc analyses. Statistical significance for the filtration pressure required to pass SS RBC through a polycarbonate filter, for the membrane shear elastic modulus and SS blood viscosity was analyzed using two-way ANOVA, followed by Dunn's multiple comparisons post hoc analyses. Statistics were calculated using GraphPad Prism 6 (San Diego, CA). Changes were considered statistically significant if $\mathrm{P}<0.05$.

\section{Results}

\subsection{GBT440 maintains RBC deformability under deoxygenated conditions}

Using RBC filterability as a surrogate for RBC deformability, we evaluated the effect of GBT440 on the deformability of SS RBCs under deoxygenated conditions in three independent experimental systems, which tested: 1) the ability of SS RBCs to move through a gel filtration column (Fig. 1A), 2) the pressure required to enable SS RBCs to pass through a polycarbonate filter (Fig. 1B) and 3) the membrane shear elastic modulus of SS RBCs via micropipette aspiration. GBT440 concentrations of $0.5,1.0$, and $2 \mathrm{mM}$ decreased the P50 (oxygen partial pressure at which $50 \%$ of the $\mathrm{Hb}$ is saturated with oxygen) of SS blood (at $20 \% \mathrm{Hct}$ ) from $31 \mathrm{mmHg}$ to 20,6 and $5 \mathrm{mmHg}$, respectively.

As shown in Figure 2A, both AA RBCs and SS RBCs moved efficiently through the gel filtration column under oxygenated conditions demonstrating the ability to deform. Similarly, AA RBCs moved efficiently through the gel filtration column under deoxygenated conditions $\left(2 \% \mathrm{O}_{2}\right)$ (Fig. 2A). In contrast, SS RBCs did not efficiently move through the gel filtration column under deoxygenated conditions indicating a loss of deformability due to deoxy-HbS polymerization under these conditions. However, GBT440-treated SS RBCs efficiently moved through the gel filtration column demonstrating improved deformability under deoxygenated conditions (Fig. 2A and B).

Compared to deformable RBCs, higher pressures are required to pass non-deformable RBCs through a polycarbonate filter. As shown in Figure 3, both AA RBCs and AS RBCs remain deformable under deoxygenated conditions, requiring a small change in pressure drop across the filter up to flow rates of $900 \mu \mathrm{L} / \mathrm{min}$ (Fig. 3). In contrast to the later, much higher pressures were required to pass deoxygenated SS RBCs through the filter, indicating a loss of SS RBC deformability. However, relative to untreated SS RBCs, GBT440-treated SS 
RBCs $(0.5 \mathrm{mM}$ and $1 \mathrm{mM})$ required reduced the pressure to pass through the filter under deoxygenated conditions (Fig. 3).

In micropipette aspiration experiments, low membrane shear elastic modulus based on simultaneous measurements of forces and deformations exerted on a red blood cell, indicates the contribution of cell membrane to RBC deformability and blood viscosity. Deformable RBCs show low shear elastic modulus $\left(<40 \mathrm{mDynes} / \mathrm{cm}^{2}\right)$, whereas rigid or less deformable RBCs show high shear elastic modulus $\left(>40 \mathrm{mDynes} / \mathrm{cm}^{2}\right)$. As shown in Figure 4, both AA RBCs and AS RBCs remained deformable under deoxygenated conditions $\left(\mathrm{pO}_{2}\right.$ of $30 \mathrm{~mm}$ $\mathrm{Hg}$ ). In contrast, under deoxygenated conditions, SS RBCs were much less deformable compared to AA RBCs (or AS RBCs) as indicated by the relatively higher shear elastic modulus (Fig. 4). However, GBT440 improved SS RBC deformability in this assay as indicated by the relatively reduced elastic modulus in GBT440-treated SS RBCs versus untreated SS RBCs under deoxygenated conditions (Fig. 4). These results indicate that GBT440 treatment restores deformability to SS RBCs to a similar degree as observed with AS RBCs. Thus, results from three independent in vitro deformability assays demonstrate that inhibition of $\mathrm{HbS}$ polymerization by GBT440 maintains SS RBC deformability under deoxygenated conditions.

\subsection{GBT440 reduces the hyperviscosity of SS blood under deoxygenated conditions}

Normal human blood exhibits characteristic shear thinning (non-Newtonian) behavior in which the measured viscosity decreases with increasing shear rate. This behavior is primarily due to RBC-RBC adhesive interactions at lower shear rates; at higher shear rates, the greater fluid forces counter their adhesive interactions thereby reducing the viscosity [23]. We used a cone/plate viscometer to determine the effects of GBT440 on SS blood viscosity at different shear rates under deoxygenated conditions and compared it to SS and AA blood viscosity under oxygenated and deoxygenated conditions. GBT440 concentrations of $0.6,1.4$, and $1.6 \mathrm{mM}$ decreased the P50 of SS blood (at 30\% Hct) from $30 \mathrm{mmHg}$ to 24 , 5.5 and $5 \mathrm{mmHg}$, respectively. In general, the viscosity of SCD blood [oxygenated (oxy SS) or deoxygenated (deoxy SS)] decreased with increasing shear rates (Fig. 5A and B). However, the viscosity of deoxy SS blood was higher than that of oxy SS blood at all shear rates evaluated, most likely due to the presence of HbS polymers in deoxygenated SS RBCs. GBT440 dose-dependently decreased the hyperviscosity of deoxy SS blood ( $\mathrm{N}=3$ patients), suggesting that GBT440 reduced the HbS polymer load within SS RBCs under deoxygenated conditions. Of note, at approximately equimolar concentrations of GBT440 $(1.4 \mathrm{mM})$ and $\mathrm{HbS}(1.5 \mathrm{mM})$ in SS blood $(30 \% \mathrm{Hct})$, the viscosity profiles of GBT440modified deoxy SS blood and unmodified oxy SS blood were similar (Fig. 5A and B).

For a more robust evaluation, we subsequently measured the effect of GBT440 $(1.6 \mathrm{mM})$ on the viscosity of SCD blood (30\% Hct) from eleven patients under both oxygenated and deoxygenated conditions. Blood from healthy volunteers (AA blood) was used as a control for comparison. As shown in Figure 5C, the viscosity of SS blood was in general higher than that of AA blood under both oxygenated and deoxygenated conditions. The difference in viscosity observed under oxygenated conditions between SS RBCs and AA RBCs may be due to cell membrane/cytoskeleton damage in SS RBCs because of $\mathrm{HbS}$ polymerization and 
sickling. Consistent with the previous finding in Figure 5, GBT440 reduced the hyperviscosity of deoxy SS blood to that of oxy SS blood (Fig. 5C). Surprisingly, GBT440 also significantly reduced the viscosity of oxy SS blood relative to untreated oxy SS blood control (Fig. 5D).

\section{Discussion}

In SCD, HbS polymer load and RBC membrane and cytoskeleton damage causes abnormal blood rheology which contributes to vasoocclusion. Hyperviscosity, a hallmark of SCD, leads to reduced blood flow, anemia and impaired oxygen delivery. Painful vasoocclusion, acute chest syndrome and osteonecrosis have been considered part of the phenotype of vascular occlusion in primarily post-capillary venules. Epidemiological studies have demonstrated that increased viscosity is an important component of SCD. Elevated blood viscosity in sickle cell patients seems to predispose this population to proliferative vascular problems [24]. Prevention of deoxygenation with GBT440 could prevent the decrease on RBC deformability and preserve normal blood flow and tissue perfusion. Prevention of hyperviscosity and of RBC deformability could decrease vasoocclusive events. While RBC deformability is not a predictor of the occurrence of vasoocclusive events in sickle cell patients, reduced RBC deformability seems to increase the risks for retinopathy in this population [25]. Using RBC filterability as a surrogate for RBC deformability, we demonstrated that GBT440 improves the deformability of SS RBCs under deoxygenated conditions in three independent in vitro assays. GBT440 enabled efficient movement of SS RBCs through a gel filtration resin, reduced the pressure required to pass SS RBCs through a polycarbonate filter and the shear elastic modulus of the RBC membrane via aspiration of SS RBCs into a micropipette under deoxygenated conditions. In addition, GBT440 dosedependently reduced the viscosity of SS blood under deoxygenated conditions. In the presence of GBT440 (1.4 and $1.6 \mathrm{mM})$ the viscosity of deoxy SS blood $(4.3 \mathrm{cP}$, at shear rates ranging from $60-415 \mathrm{~s}^{-1}$ and $37^{\circ} \mathrm{C}$ ) was similar to that of untreated oxy SS blood (3.9 $\mathrm{cP})$. Considering the duration of deoxygenation ( $>30 \mathrm{~min}$ ) in our assays is significantly greater than the typical HbS polymerization delay time $(<1 \mathrm{~min})$ [26], SS RBCs are expected to contain $\mathrm{HbS}$ polymers. Therefore, the observed improvements in deformability and viscosity afforded by GBT440 are most likely due to the reduction of HbS polymer load at steady state. This may explain why high GBT440 concentrations were required to demonstrate improvements in both deformability and viscosity under the in vitro deoxygenations conditions used herein. Interestingly, GBT440 also reduced the viscosity of SS blood under room air conditions. As SS blood is expected to contain very little to no $\mathrm{HbS}$ polymers at room air [27], these data suggest that perhaps in addition to its antipolymerization activity, GBT440 may mitigate SS RBC viscosity via an alternative mechanism. In previous work, the heterocyclic aldehyde 5-hydroxymethyl-2-furfural (5HMF), an $\mathrm{Hb}-\mathrm{O}_{2}$ affinity modulator, was found to react with the cation transport systems in RBCs leading to increased red cell hydration and reduced cytoplasmic viscosity [28]. Thus, as an aldehyde, GBT440 could have a similar effect as observed with 5HMF on the transport systems in RBCs. However, the later remains to be determined. Patients with sickle cell disease are characterized by the presence of dense erythrocytes, which are formed because of cell dehydration and potassium loss, in a phenomenon called Psickle activation 
[29]. Future studies will explore the role of RBC oxygenation in Psickle activation, as it is expected that preventing deoxygenation of SS $\mathrm{Hb}$ with GBT440, would reduce sickling and the activation of the Gardos channel, and reduce erythrocyte dehydration.

Of important note, the use of citrate as anticoagulant in this study is not recommended by the Hemorheological Laboratory Techniques for viscosity measurements, as citrate solution dilutes the blood [30]. Unfortunately, the approved blood collection protocol did not include ethylenediaminetetraacetate (EDTA) or heparin. Hematocrit variations due to blood dilutions with citrate solution affect the absolute value of the blood viscosity. However, the relative changes between treated and untreated under normoxia and hypoxia remain valid, as all samples were collected using citrate. Other parameters besides blood viscosity are less affected by the blood collection in citrate, since hematocrits were drastically decreased using appropriate buffers to measure filterability and membrane elastic modulus. The RBCs need to undergo considerable deformations, to pass through the microcirculation. The increased rigidity of SS RBCs, impart due to the intra-cellular sickle $\mathrm{Hb}$ polymers, exhibits a substantial increase in the blood flow resistance and potentially triggers vaso-occlusion in the microcirculation.

Besides altering the cell rigidity, the growing SS Hb polymer domain inside the SS RBC can potentially distort ("sickle") the cell membrane, and therefore change the cell morphology under certain conditions. Several techniques have recently been developed to measure deformability of the RBC. Ektacytometry is the goal standards for RBC deformability; however, ektacytometers do not allow for the control of the oxygenation of the samples. While the cell deformability depends on the intracellular mean corpuscular $\mathrm{Hb}$ concentration values, the cell preparation process such as oxygenation can affect the intracellular polymer configuration and therefore affect the cell morphologies. Due to an initial lack of suitable instrumentation, to study RBC deformability and aggregation under normoxic and hypoxic conditions, subrogates indictor of RBC deformability were used in this study.

In conclusion, our studies suggest that by inhibiting upstream $\mathrm{HbS}$ polymerization and thus preserving SS RBC health, GBT440 may provide clinical benefit by preventing SS RBC sickling, improving SS RBC deformability and reducing the hyperviscosity of blood in SCD patients. Ongoing clinical investigation will determine whether these observed rheological and hemodynamic improvements manifest as improved clinical outcomes.

\section{Acknowledgements}

We will like to acknowledge the University of North Carolina Comprehensive Sickle Cell Program (UNC, Chapel Hill, NC) for providing blood from sickle cell patients.

Competing interests: Kobina Dufu, Mira P. Patel and Donna Oksenberg are employees and shareholders of Global Blood Therapeutics, Inc. Pedro Cabrales was funded by Global Blood Therapeutics.

\section{References}

[1]. Bunn HF. Pathogenesis and treatment of sickle cell disease. N Engl J Med. 1997;337(11):762-9. [PubMed: 9287233]

[2]. Eaton WA, Hofrichter J. Sickle Cell Hemoglobin Polymerization. Adv Protein Chem. 1990;40(C): 63-279. [PubMed: 2195851] 
[3]. Ferrone F Polymerization and Sickle Cell Disease: A Molecular View. Microcirculation. 2004;11(2):115-28. Available from: http://doi.wiley.com/10.1080/10739680490278312 [PubMed: 15280087]

[4]. Kim Y, Kim K, Park Y. Measurement Techniques for Red Blood Cell Deformability: Recent Advances. 1979.

[5]. Dong C, Chadwick RS, Schechter AN. Influence of sickle hemoglobin polymerization and membrane properties on deformability of sickle erythrocytes in the microcirculation. Biophys J. 1992;63(3):774-83. [PubMed: 1420913]

[6]. Rees DC, Williams TN, Gladwin MT. Sickle-cell disease. Lancet. 2010;376(9757):2018-31. [PubMed: 21131035]

[7]. Wagner MC, Eckman JR, Wick TM. Sickle cell adhesion depends on hemodynamics and endothelial activation. J Lab Clin Med. 2004;144(5):260-7. [PubMed: 15570244]

[8]. Barabino GA, Platt MO, Kaul DK. Sickle Cell Biomechanics. Annu Rev Biomed Eng. 2010;12(1): 345-67. [PubMed: 20455701]

[9]. Ballas SK, Smith ED. Red Blood Cell Changes During the Evolution of the Sickle Cell Painful Crisis. Blood. 1992;79(8):2154-63. [PubMed: 1562742]

[10]. Embury SH. The Not-So-Simple Process of Sickle Cell Vasoocclusion. Microcirculation. 2004;11:101-13. [PubMed: 15280086]

[11]. Connes P, Alexy T, Detterich J, Romana M, Hardy-Dessources MD, Ballas SK. The role of blood rheology in sickle cell disease. Blood Reviews. 2016;30:111-18. [PubMed: 26341565]

[12]. Bowers AS, Duncan WW, Pepple DJ. Red blood cell deformability is reduced in homozygous sickle cell disease patients with leg ulcers. Clinical Hemorheology and Microcirculation. 2016;64:199-204. [PubMed: 27258199]

[13]. Charlot K, Romana M, Moeckesch B, Jumet S, Waltz X, Davialle-Doumdo L, et al. Which side of the balance determines the frequency of vaso-occlusive crises in children with sickle cell anemia: Blood viscosity or microvascular dysfunction? Blood Cells, Molecules and Diseases. 2016;56:41-45.

[14]. Li X, Du E, Lei H, Tang YH, Dao M, Suresh S, Karniadakis GE. Patient-specific blood rheology in sickle-cell anaemia. Interface Focus. 2016;6(1):20150065. Available from 10.1098/rsfs. 2015.0065

[15]. Ballas S Sickle cell anemia with few painful crises is characterized by decreased red cell deformability and increased number of dense cells. Am J Hematol. 1991;26(2):122-30.

[16]. Oksenberg D, Dufu K, Patel MP, Chuang C, Li Z, Xu Q, et al. GBT440 increases haemoglobin oxygen affinity, reduces sickling and prolongs RBC half-life in a murine model of sickle cell disease. Br J Haematol. 2016;175(1):141-53. [PubMed: 27378309]

[17]. Brugnara C, Van Ha T, Tosteson DC. Acid pH induces formation of dense cells in sickle erythrocytes. Blood. 1989;74:487-95. [PubMed: 2752126]

[18]. Pais E, Cambridge JS, Johnson CS, Meiselman HJ, Fisher TC, Alexy T. A novel high-throughput screening assay for sickle cell disease drug discovery. J Biomol Screen. 2009;14(4):330-6. [PubMed: 19403916]

[19]. Cabrales P Effects of erythrocyte flexibility on microvascular perfusion and oxygenation during acute anemia. AJP Hear Circ Physiol. 2007;293(2):H1206-15.

[20]. Skalak R, Impelluso T, Schmalzer EA, Chien S. Theoretical modeling of filtration of blood cell suspensions. Biorheology. 1983;20(1):41-56. [PubMed: 6871425]

[21]. Evans EA. Bending elastic modulus of red blood cell membrane derived from buckling instability in micropipet aspiration tests. Biophys J. 1983;43(1):27-30. [PubMed: 6882860]

[22]. Baskurt OK, Meiselman HJ. Blood Rheology and Hemodynamics. Seminars in Thrombosis and Hemostasis. 2003 p. 435-50. [PubMed: 14631543]

[23]. Alexy T, Pais E, Armstrong JK, Meiselman HJ, Johnson CS, Fisher TC. Rheologic behavior of sickle and normal red blood cell mixtures in sickle plasma: Implications for transfusion therapy. Transfusion. 2006;46(6):912-8. [PubMed: 16734807]

[24]. Lemaire C, Lamarre Y, Lemmone N, Waltz X, Chahed S, Cabot F, et al. Severe proliferative retinopathy is associated with blood hyperviscosity in sickle cell hemoglobin-C disease but not in sickle cell anemia. Clin Hemorheol Microcirc. 2013;55(2): 205-12. [PubMed: 23076002] 
[25]. Lemonne N, Lamarre Y, Romana M, Hardy-Dessources M- D, Lionnet F, Waltz X, et al. Impaired blood rheology plays a role in the chronic disorders associated with sickle cellhemoglobin C disease. Haematologica. 2014;99(5):74-75. [PubMed: 24633868]

[26]. Li Q, Henry ER, Hofrichter J, Smith JF, Cellmer T, Dunkelberger EB, et al. Kinetic assay shows that increasing red cell volume could be a treatment for sickle cell disease. PNAS. 2017;114(5):E689-96. [PubMed: 28096387]

[27]. Noguchi CT, Torchia DA, Schechter AN. Determination of deoxyhemoglobin S polymer in sickle erythrocytes upon deoxygenation. PNAS. 1980;77(9):5487-91. [PubMed: 6933568]

[28]. Hannemann A, Cytlak UM, Rees DC, Tewari S, Gibson JS. Effects of 5-hydroxymethyl-2furfural on the volume and membrane permeability of red blood cells from patients with sickle cell disease. J Physiol. 2014;592(18).

[29]. Ranney HM. 1997. Psickle, the temporary leaky link between sickling and cellular dehydration. J Clin Invest. 1997;99(11):2559-60. [PubMed: 9169482]

[30]. Baskurt OK, Boynard M, Cokelet GC, Connes P, Cooke BM, Forconi S, et al. New guidelines for hemorheological laboratory techniques. Clinical hemorheology and microcirculation. 2009;42(2): 75-97. [PubMed: 19433882] 
A

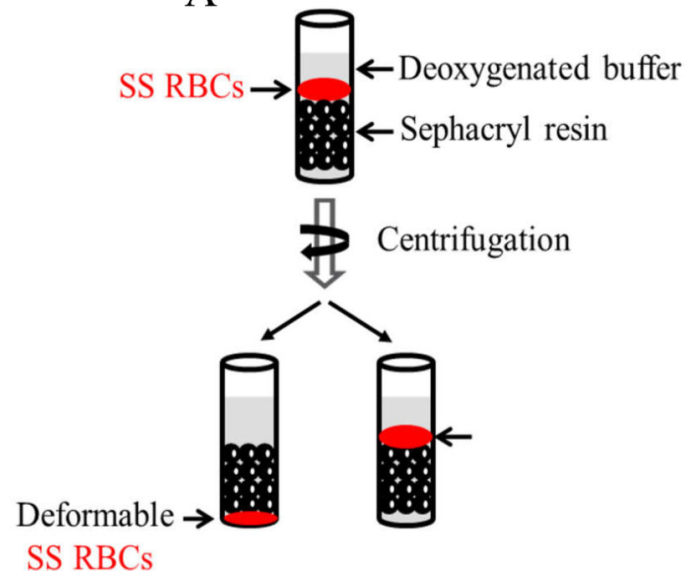

B

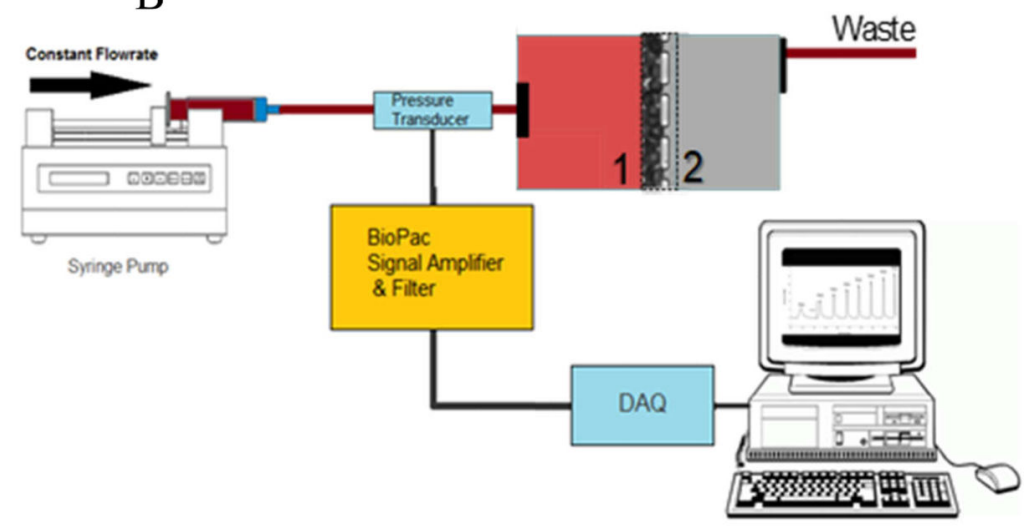

Fig. 1.

Schematic of the two filterability assays. A) Red blood cell deformability was assessed via a modified gel filtration. Deformable RBCs were separated from non-deformable RBCs, and lysed to determine the relative concentration of $\mathrm{Hb}$ in deformable RBCs and non-deformable RBC fractions. B) Red blood cell deformability was also measured using the cellular filtration through a membrane filter with $5.0 \mu \mathrm{m}$ pores size. Deformability was determined based on the pressure required to maintain specific flow rates. 
A
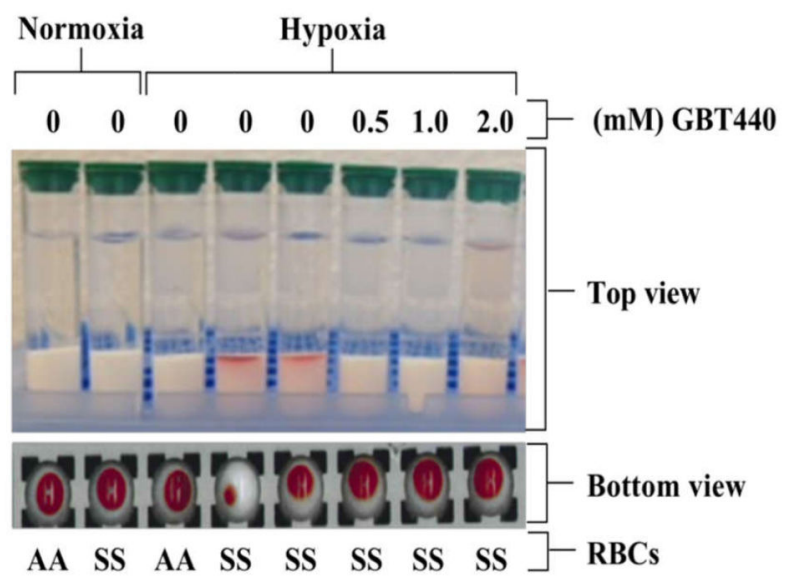

B

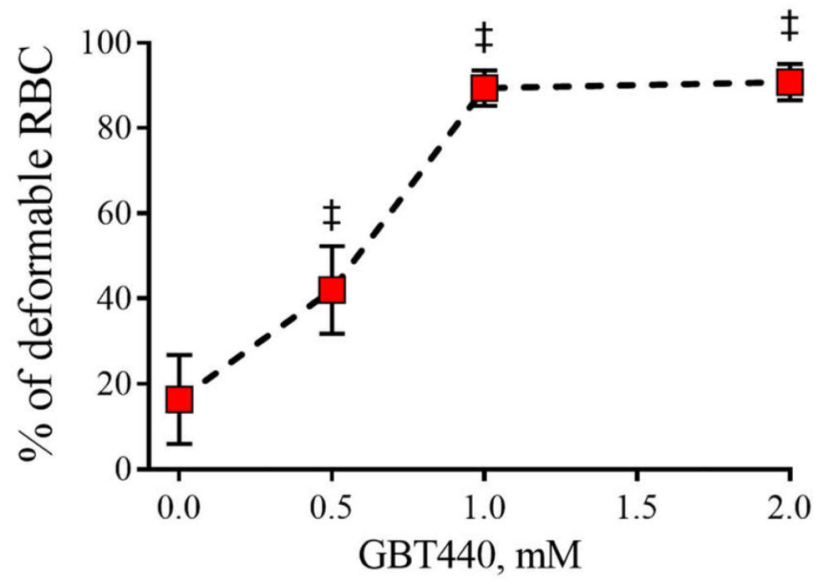

Fig. 2.

GBT440 enables movement of SS RBCs through a gel filtration resin under deoxygenated conditions. RBCs were centrifuged through a gel filtration column under deoxygenated conditions $\left(2 \% \mathrm{O}_{2} \sim \mathrm{pO}_{2}\right.$ of $\left.16 \mathrm{~mm} \mathrm{Hg}\right)$. A) Deformable RBCs moved to the bottom of the gel filtration resin upon centrifugation, whereas non-deformable RBC remained at the top of the gel filtration resin upon centrifugation. B) Fraction of deformable RBCs was calculated based on the amount of $\mathrm{Hb}$ in the deformable and non-deformable fractions of RBCs.

Hemoglobin in each fraction were quantified by measuring absorbance at $542 \mathrm{~nm}$ and 700 $\mathrm{nm}$.. Data obtained from one in three replicates. Final concentration of DMSO was $2 \%$. $\ddagger, P$ $<0.01$ compared to without GBT440. 


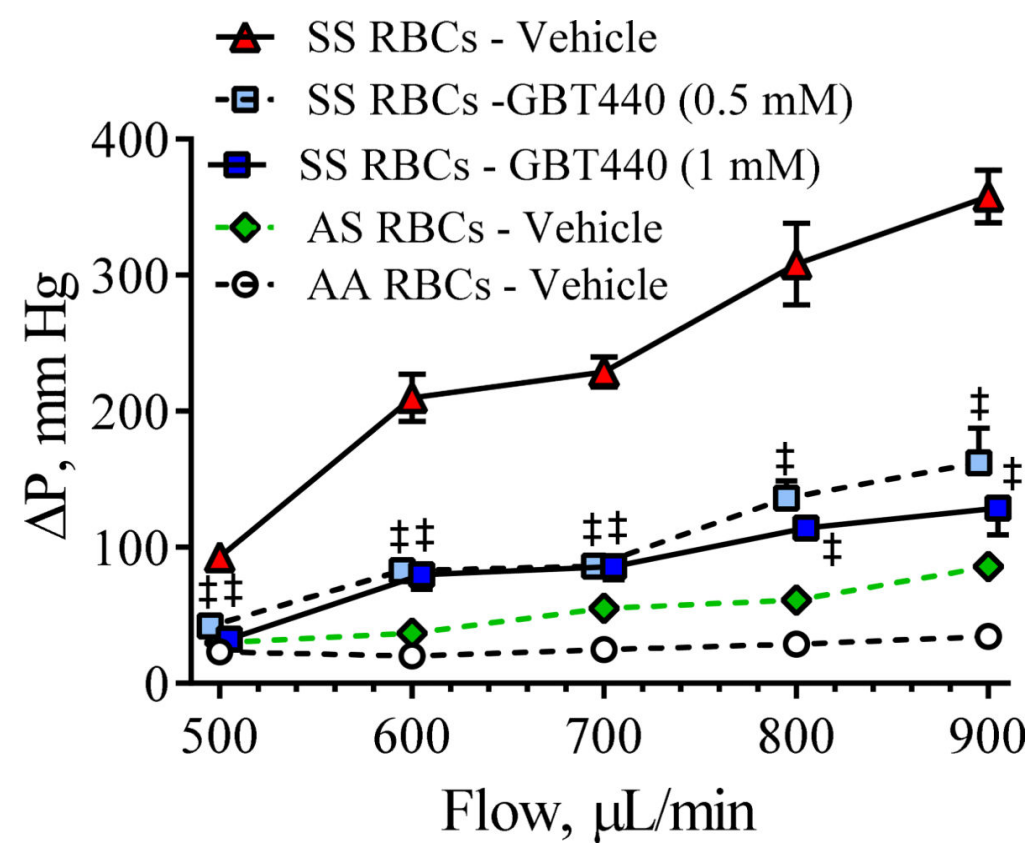

Fig. 3.

GBT440 reduces the pressure required to pass SS RBCs through a polycarbonate filter under deoxygenated conditions. A) Schematic showing thin film deoxygenation of blood. A deoxygenated-RBC suspension was passed through a polycarbonate filter (shown in B) at various flow rates $(0.5,0.6,0.7,0.8,0.9$ and $1.0 \mathrm{~mL} / \mathrm{min})$ and differential pressure drop across the filter was measured. RBCs were pre-incubated with GBT440 or DMSO control prior to deoxygenation. C) Quantification of the change in pressure across the polycarbonate filter per flow rate. Mean $\pm \mathrm{SD}$ are shown, $\mathrm{N}=1$ donor, 5 replicates. The final concentration of DMSO was $1.25 \%$. $\ddagger$ P $<0.01$ compared to without GBT440. 


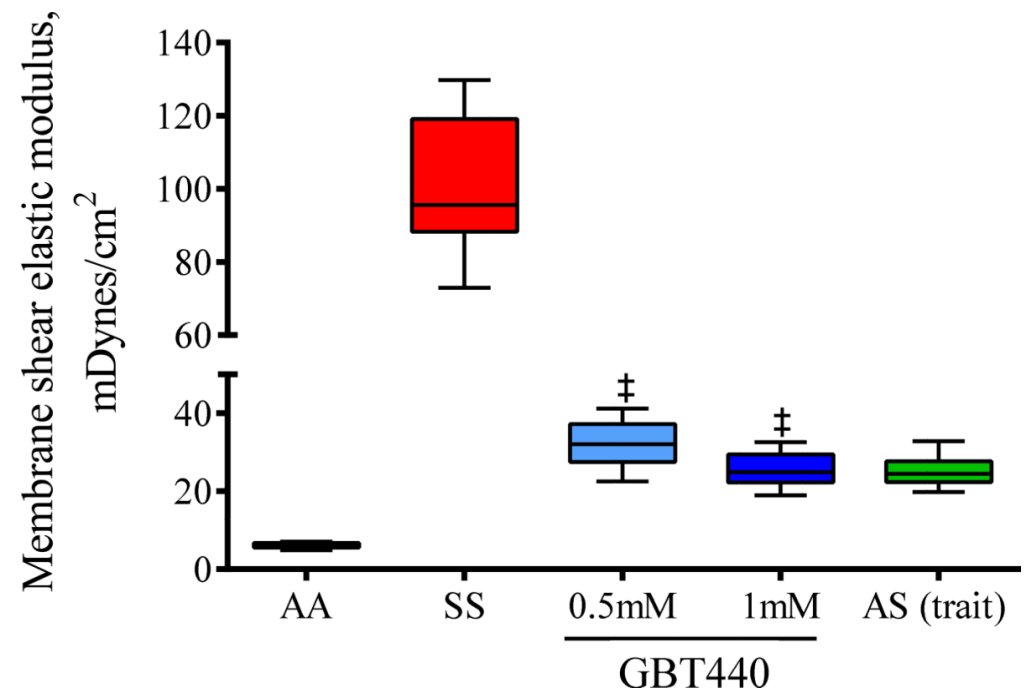

Fig. 4.

GBT440 reduces the tension required to aspirate SS RBCs under deoxygenated conditions. A) Schematic of micropipette aspiration of RBCs. L denotes aspiration length, P denotes aspiration pressure and Rp denotes internal radius of pipette. Deoxygenated-RBCs were aspirated into a micropipette with an internal diameter of $\sim 2$ micron. Cell membrane elastic module and cell volume were calculated based on the relationship between pressure and aspiration length. B) Membrane shear elastic modulus (tension) required to aspirate RBCs into the micropipette. RBCs were pre-incubated with GBT440 or DMSO prior to deoxygenation. The final concentration of DMSO was $1.25 \%$. Mean \pm SD are shown, $\mathrm{N}=1$ donor, 3 replicates. $\ddagger$ P $<0.01$ compared to without GBT440. 

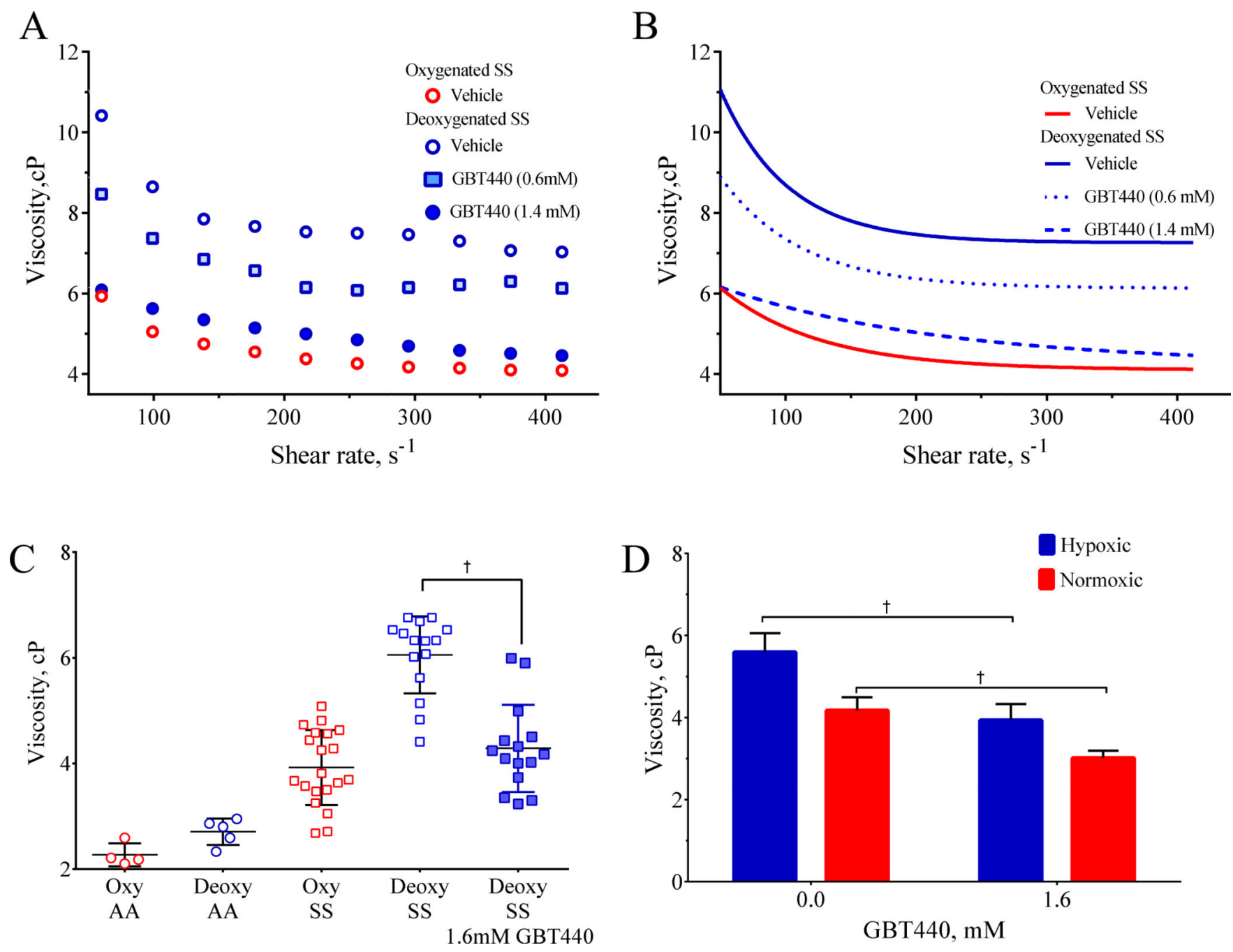

Fig. 5.

GBT440 reduces the hyperviscosity observed in SS blood under oxygenated and deoxygenated conditions. A) Blood viscosity (measured as Centipoise, $\mathrm{cP}$ ) was determined at various shear rates under oxygenated and deoxygenated conditions. The graph is a representative experiment from a total of 3 different patient blood samples. B) Sickle cell blood viscosity $(\mathrm{N}=11)$ was determined at various shear rates under oxygenated and deoxygenated conditions. The $\mathrm{cP}$ median $\pm \mathrm{SEM}$ is shown for each sample. The data for deoxy SS and deoxy SS + GBT440 represent 15 individual experiments with 11 different patient blood samples. C) Sickle cell blood viscosity was determined under oxygenated and deoxygenated conditions. Results are expressed as $\mathrm{cP}$ mean $\pm \mathrm{SEM}$; $\mathrm{p}$ values were obtained from a two-tailed paired t test. Data represents 7 individual experiments with 3 different patient blood samples. Sickle cell blood was pre-incubated with GBT440 (1.6 mM) or DMSO prior to deoxygenation. The final concentration of DMSO was $2 \%$. $\dagger, \mathrm{P}<0.05$ compared to without GBT440. 